\title{
SORÇÃO, DESSORÇÃO E POTENCIAL DE LIXIVIAÇÃO DE ATRAZINE EM SOLOS BRASILEIROS
}

\author{
ELIANE REGINA ARCHANGELO ${ }^{1}$, HÉLIO TEIXEIRA PRATES ${ }^{2}$, FRANCISCO AFFONSO \\ FERREIRA $^{3}$, DÉCIO KARAM ${ }^{4}$, LINO ROBERTO FERREIRA ${ }^{5}$, ANTÔNIO AMÉRICO CARDOSO ${ }^{6}$
}

\begin{abstract}
IPesquisadora/DCR-Unitins Agro. CEP. 77020-122 Palmas,TO. E-mail: earchangelo@yahoo.com.br (autora para correspondência).

${ }^{2}$ Pesquisador da Embrapa Milho e Sorgo. Caixa postal 151, CEP. 35701-970 Sete Lagoas, MG. E-mail: htprates@cnpms.embrapa.br

${ }^{3}$ Prof. do Depto de Fitotecnia da Universidade Federal de Viçosa - UFV. Av. PH Holfs, s/n, CEP. 36571-000 Viçosa, MG.E-mail:faffonso@ufv.br

${ }^{4}$ Pesquisador da Embrapa Milho e Sorgo. Caixa postal 151, CEP. 35701-970 Sete Lagoas, MG. E-mail: karam@cnpms.embrapa.br

${ }^{3}$ Prof. do Depto de Fitotecnia da Universidade Federal de Viçosa - UFV. Av. PH Holfs, s/n, CEP. 36571-000 Viçosa, MG.E-mail:lroberto@ufv.br

${ }^{3}$ Prof. do Depto de Fitotecnia da Universidade Federal de Viçosa - UFV. Av. PH Holfs, s/n, CEP. 36571-000 Viçosa, MG.E-mail:acardoso@ufv.br.
\end{abstract}

Revista Brasileira de Milho e Sorgo, v.4, n.1, p.14-27, 2005

RESUMO - Determinar a sorção, a dessorção e o potencial de lixiviação de atrazine em diferentes solos, foram os objetivos desse trabalho. As amostras dos solos foram agitadas por $24 \mathrm{~h}$, centrifugadas e o sobrenadante filtrado e analisado por HPLC. As etapas da dessorção foram conduzidas por meio da reposição do sobrenadante removido com o mesmo volume de solução $0,01 \mathrm{M}$ de $\mathrm{CaCl}_{2}$ com agitação por $24 \mathrm{~h}$ e centrifugação, repetindo-se o processo por três vezes consecutivas, 24,48 e $72 \mathrm{~h}$, sendo o sobrenadante filtrado e analisado por HPLC. A sorção de atrazine, descrita pela isoterma de Freundlich, foi calculada pela diferença entre as concentrações adicionadas e aquelas quantificadas no HPLC. Foram calculadas as percentagens de dessorção a 24, 48 e $72 \mathrm{~h}$ pela diferença entre as quantidades sorvidas e aquelas quantificadas no HPLC. Correlações de Pearson foram feitas para se determinar a correlação entre $\mathrm{k}_{\mathrm{f}}, \mathrm{k}_{\mathrm{foc}}$ e dessorção e as principais propriedades dos solos. O coeficiente GUS foi utilizado para estimar o potencial de lixiviação. Os baixos valores encontrados para o $\mathrm{k}_{\mathrm{f}} \mathrm{da}$ atrazine indicam que esse herbicida é de pequena sorção pelos solos estudados. No perfil LVd-a a sorção se correlacionou significativa e positivamente com o teor de carbono orgânico quando analisado isoladamente. Os valores de $\mathrm{k}_{\text {foc }}$ variaram de 70,37 a 201,47 nas amostras superficiais dos solos estudados e apresentaram correlação significativa com o teor de carbono orgânico quando analisado somente o perfil do LVd-a. A avaliação do potencial de lixiviação demonstrou que a atrazine pode ser classificada como herbicida lixiviador em todos os solos analisados. O processo de dessorção foi menor que o processo de sorção até 72 horas após a aplicação. A dessorção é maior em camadas mais profundas no perfil do solo LVd-a, onde o teor de carbono orgânico é menor.

Palavras-chave: isoterma de Freundlich, $\mathrm{k}_{\mathrm{f}}, \mathrm{k}_{\mathrm{foc}}, \mathrm{GUS}$, herbicida. 


\title{
SORPTION, DESORPTION, AND LEACHING POTENTIAL OF ATRAZINE IN BRAZILIAN SOILS
}

\begin{abstract}
Atrazine was applied on soil samples for sorption and desorption determination and leaching potential in different soils. After 24 hours shaking, the soil samples were centrifuged, the supernatant filtered and then analyzed by HPLC. Desorption periods were established by substituting the removed supernatant volume in each $\mathrm{CaCl}_{2}$ solution $(0,01 \mathrm{M})$, tube shaking for 24 hours, and centrifuging. The procedure was repeated three times every 24,48 , and 72 hours, the supernatant was filtered, and analyzed by HPLC. Atrazine sorption, described by Freundlich isotherm, was calculated by differences between applied concentrations and those measured by the HPLC. Desorption percentages after 24,48 , and $72 \mathrm{~h}$ were calculated by the difference among the sorbed quantities and those measured in the HPLC. Pearson correlation was set up to determine the correlation between $\mathrm{k}_{\mathrm{f}}, \mathrm{k}_{\mathrm{foc}}$, desorption, and the main soil properties, and the GUS coefficient was used to estimate the leaching potential. The low atrazine $\mathrm{k}_{\mathrm{f}}$ values suggest that this herbicide is of low sorption in these soils. For the LVd-a profile a significant and positive correlation between sorption and organic carbon content was established in the individual analyses. The $\mathrm{k}_{\text {foc }}$ values varied from 70,37 to 201,47, in surface samples of the studied soils. They presented a significant correlation with the organic carbon content detected only when the LVd-a soil profile was analyzed. The leaching potential showed that atrazine may be classified as a leaching herbicide in all analyzed soils. Up to 72 hours after application, the desorption was weaker than the sorption. Desorption is greater in deeper LVd-a soil layers, in which organic carbon contents are lower.
\end{abstract}

Key words: Freundlich isotherm, $\mathrm{k}_{\mathrm{f}}, \mathrm{k}_{\mathrm{foc}}$, GUS, herbicide.

Atualmente, a agricultura moderna não sobrevive sem o uso dos agroquímicos. No entanto, a utilização incorreta destes compostos sintéticos, entre os quais os herbicidas, usados em maior quantidade, tem aumentado os riscos de contaminação do meio ambiente.

Dentre as triazinas, a atrazine $\{2$-cloro4(etilamino)-6-(isopropilamino)-s-triazina $\}$ é o herbicida mais utilizado na agricultura (Khan, 1980). As doses recomendadas para as culturas dependem das características físico-químicas do solo, sendo mais elevadas para solos mais argilosos ou ricos em matéria orgânica. Atrazine é pouco lixiviada em solos com teores médios e altos de argila ou matéria orgânica. Apresenta persistência média no solo de cinco a sete meses nas condições climáticas tropicais e subtropicais
(Rodrigues \& Almeida, 1998), podendo deixar resíduos nos solos que poderão comprometer o desenvolvimento de culturas sensíveis em rotação (Latachana, 1987).

Jenks et al. (1998) verificaram que atrazine ficou mais sorvida em amostras de solos nas camadas $0-30 \mathrm{~cm}$ do que em amostras abaixo de $60 \mathrm{~cm}$. A sorção da atrazine correlacionou-se positivamente com a matéria orgânica e negativamente com o pH.

A maioria das informações sobre o comportamento ambiental dos herbicidas é obtida em condições edafoclimáticas temperadas (Oliveira Júnior, 1998). Pouco se conhece sobre o comportamento desses herbicidas utilizados em milhões de hectares nos solos tropicais, principalmente no cerrado brasileiro. Assim sendo, são 
necessários estudos científicos sobre poluição ambiental, efeitos sobre a fauna e a flora, persistência nos diferentes solos, bem como a mobilidade destes no perfil do solo.

O desenvolvimento de estudos de sorção, dessorção e lixiviação de princípios ativos destes herbicidas favorecerá a avaliação de riscos destas substâncias nas condições edafoclimáticas do agroecossistema das culturas em condições brasileiras.

O objetivo desse trabalho foi o de estudar a sorção, a dessorção e o potencial de lixiviação da atrazine em amostras de solos brasileiros.

\section{Material e Métodos}

\section{Solos Estudados}

Os resultados das análises químicas e granulométricas dos solos estudados e suas classificações estão apresentados na Tabela 1.

Amostras de solos coletadas da camada arável $(0-15 \mathrm{~cm})$ em área da Universidade de São Paulo, campus “Luiz de Queiroz”, no município de Piracicaba-SP, classificados como Latossolo Vermelho distróférico (LVdf), textura argilosa, Latossolo Vermelho distrófico (LVd-m), textura média e Gleissolo Melânico distrófico (GMd), textura média. Também foram usadas amostras de um Latossolo Vermelho distrófico (LVd-a), textura argilosa, amostrado na Embrapa Milho e Sorgo, município de Sete Lagoas - MG. Nesse último, foi aberto um perfil onde foram coletadas amostras de $15 \mathrm{em} 15 \mathrm{~cm}$, até a profundidade de $120 \mathrm{~cm}$.

\section{Sorção e Dessorção}

Para quantificar a sorção e dessorção, utilizaram-se as relações solo:solução determinadas preliminarmente. Estas relações foram de 1:1 para LVdf e LVd-m, de 1:2 para LVd-a e de 1:8 para GMd. As amostras para os estudos da atrazine foram $10 \mathrm{~g}$ dos solos LVdf, LVd-m, $5 \mathrm{~g}$ LVd-a e de 1,25 g para GMd. Estas foram colocadas em tubos de centrífuga com capacidade de $50 \mathrm{~mL}$. Nesses tubos foram adicionados $10 \mathrm{~mL}$ da solução do herbicida com 99,0 \% de pureza. As concentrações utilizadas foram de 0,125 ; 0,250; 0,500; 1,000; 2,000 e 4,000 $\mu \mathrm{g} \mathrm{mL}^{-1}$. Essas concentrações foram preparadas em solução $0,01 \mathrm{M} \mathrm{de} \mathrm{CaCl}_{2}$, com duas repetições. O delineamento experimental utilizado foi o inteiramente casualizado.

Os tubos foram então fechados com tampas rosqueáveis, agitados em misturador vórtex por 10 segundos, e levados a um agitador horizontal por 24 horas a $200 \mathrm{rpm}$, à temperatura ambiente $\left(27 \pm 2{ }^{\circ} \mathrm{C}\right)$. Ao final desse período, os tubos foram centrifugados por 10 min a $2200 \mathrm{rpm}$. O sobrenadante recolhido foi passado em filtro de membrana PVDF de 0,45 $\mu \mathrm{m}$ e injetado no HPLC (Cromatografia Líquida de Alta Eficiência). A retenção do herbicida pelas membranas dos filtros foi menor que $1 \%$.

As etapas para determinar a dessorção foram conduzidas por meio da reposição do sobrenadante removido, pelo mesmo volume de solução $0,01 \mathrm{M}$ de $\mathrm{CaCl}_{2}$ em cada tubo. Completado o volume, os tubos foram então fechados, agitados em misturador vórtex por 10 segundos e levados ao agitador horizontal por 24 horas a $200 \mathrm{rpm}$, à temperatura ambiente de $27 \pm 2{ }^{\circ} \mathrm{C}$. As etapas de retirada do sobrenadante, reposição, nova suspensão da mistura solo:solução e reequilíbrio no agitador horizontal foram repetidas três vezes, 24, 48 e 72 horas, consecutivamente. O sobrenadante recolhido foi passado em filtro de membrana PVDF de $0,45 \mu \mathrm{m}$ e injetado no HPLC para determinar as dessorções.

Procedimentos para Determinação da Concentração Sorvida (Cs), da Concentração de Equilíbrio (Ce), do Coeficiente de Sorção $\left(\mathrm{k}_{\mathrm{f}}\right)$, 
TABELA 1. Principais características químicas e granulométricas ${ }^{1 /}$ dos solos estudados.

\begin{tabular}{|c|c|c|c|c|c|c|c|c|}
\hline \multirow{2}{*}{$\begin{array}{l}\text { Classes }^{2 /} \\
\text { dos solos }\end{array}$} & \multirow{2}{*}{$\begin{array}{l}\text { Prof. } \\
(\mathrm{cm})\end{array}$} & \multirow{2}{*}{ pH } & \multirow{2}{*}{$\begin{array}{c}\mathrm{CO} \\
\text { dag } \mathrm{kg}^{-1}\end{array}$} & \multirow{2}{*}{$\begin{array}{c}\mathrm{CTC} \\
\mathrm{Cmol}_{\mathrm{c}} \mathrm{dm}^{-3}\end{array}$} & SB & Argila & Silte & Areia \\
\hline & & & & & \multicolumn{4}{|c|}{$\%$} \\
\hline LVd-a & $0-15$ & 5,3 & 2,41 & 8,44 & 22,99 & 73 & 18 & 9 \\
\hline LVd-a & $15-30$ & 5,0 & 1,91 & 8,37 & 8,36 & 73 & 18 & 9 \\
\hline LVd-a & $30-45$ & 5,0 & 1,65 & 8,34 & 4,08 & 73 & 17 & 10 \\
\hline LVd-a & $45-60$ & 5,2 & 1,40 & 6,83 & 3,81 & 74 & 16 & 10 \\
\hline LVd-a & $60-75$ & 5,2 & 1,23 & 6,88 & 2,47 & 69 & 22 & 9 \\
\hline LVd-a & $75-90$ & 5,2 & 1,11 & 6,34 & 1,89 & 75 & 16 & 8 \\
\hline LVd-a & $90-105$ & 5,2 & 1,14 & 6,11 & 1,64 & 72 & 20 & 8 \\
\hline LVd-a & $105-120$ & 5,2 & 1,07 & 5,58 & 1,43 & 75 & 16 & 9 \\
\hline LVdf & $0-15$ & 5,1 & 1,62 & 10,15 & 30,64 & 68 & 13 & 19 \\
\hline LVd-m & $0-15$ & 6,6 & 0,68 & 8,31 & 80,99 & 22 & 04 & 74 \\
\hline GMd & $0-15$ & 4,5 & 8,53 & 21,76 & 18,20 & 44 & 45 & 11 \\
\hline
\end{tabular}

1/ Análises realizadas na Embrapa Milho e Sorgo, Sete Lagoas-MG.

2/ LVd-a, Latossolo Vermelho distrófico, textura argilosa; LVdf, Latossolo Vermelho distroférico, textura argilosa; LVd-m, Latossolo Vermelho distrófico, textura média; GMd, Gleissolo Melânico distrófico, textura média.

Prof.: Profundidade de amostragem; CO: Carbono Orgânico; CTC: Capacidade de Troca Catiônica; SB: Saturação de Bases.

do Índice da Intensidade de Sorção (1/n), do Coeficiente de Sorção Normalizado para o Carbono Orgânico $\left(\mathrm{k}_{\text {foc }}\right)$, do Potencial de Lixiviação (GUS) e das Dessorções e Análise dos Dados.

A concentração sorvida (Cs), expressa em $\mathrm{mg} \mathrm{kg}^{-1}$ solo, foi calculada pela diferença entre a concentração da solução inicial ou adicionada $\left(\mathrm{Ci}, \mathrm{mg} \mathrm{L}^{-1}\right)$ e a concentração da solução em equilíbrio com o solo $\left(\mathrm{Ce}, \mathrm{mg} \mathrm{L}^{-1}\right): \mathbf{C s}=(\mathbf{C i}-\mathbf{C e})$ (V/M), onde V é o volume de solução (L) e M, quantidade de solo $(\mathrm{kg})$.

A partir da concentração de equilíbrio (Ce) e da quantidade sorvida (Cs), determinadas experimentalmente, foram ajustadas as isotermas de Freundlich, utilizando-se a equação: $\mathbf{C s}=\mathbf{k}_{\mathbf{f}}$ $\mathbf{C e}^{1 / \mathrm{n}}$ em que $\mathrm{k}_{\mathrm{f}}=$ coeficiente de sorção e $1 / \mathrm{n}=$ índice da intensidade da sorção, que indica o grau de linearidade da isoterma.

A constante de sorção normalizada para o carbono orgânico $\left(\mathrm{k}_{\text {foc }}\right)$ foi calculada utilizando-se $k_{\mathrm{f}}$ na expressão: $\mathbf{k}_{\text {foc }}=\left(\mathbf{k}_{\mathrm{f}} / \mathbf{\%} \mathbf{C O}\right) \mathbf{} \mathbf{1 0 0}$. Para a cla sificação dos herbicidas quanto ao seu potencial de lixiviação, utilizou-se o índice proposto por Gustafson (1989): GUS $=\log t_{1 / 2}(4-$ $\left.\log \mathbf{k}_{\text {foc }}\right)$.

$\mathrm{O}$ resultados de $\mathrm{k}_{\mathrm{f}}$ em função dos teores de carbon orgânico foram submetidos às análises de váriância e de regressão, ajustando-se 
modelos polinomiais de $1^{\circ}, 2^{\circ}$ e $3^{\circ}$ graus. Foi selecionada a equação de regressão de efeito significativo pelo teste $\mathrm{F}$ a $5 \%$ de probabilidade e de significado agronômico.

As concentrações de atrazine presentes

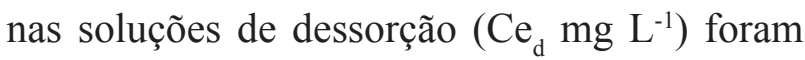
determinadas e a quantidade de cada herbicida que permaneceu sorvida ao solo após cada passo de dessorção (Csdes ${ }_{24 \mathrm{~h}}$; Csdes $_{48 \mathrm{~h}}$ e Csdes $_{72 \mathrm{~h}} \mathrm{mg} \mathrm{kg}^{-}$ $\left.{ }^{1}\right)$ foi calculada por diferença, conforme segue:

$$
\begin{gathered}
\text { Csdes }_{24 \mathrm{~h}}=\left(\mathrm{Cs}-\mathrm{Ce}_{\mathrm{d}}\right) \\
\text { Csdes }_{48 \mathrm{~h}}=\left(\operatorname{Csdes}_{24 \mathrm{~h}}-\mathrm{Ce}_{\mathrm{d}}\right) \\
\operatorname{Csdes}_{72 \mathrm{~h}}=\left(\operatorname{Csdes}_{48 \mathrm{~h}}-\mathrm{Ce}_{\mathrm{d}}\right)
\end{gathered}
$$

Em seguida, foi calculada a percentagem de dessorção a cada nível de diluição e os dados, submetidos à análise de variância e de regressão linear.

Os resultados da percentagem de dessorção em função dos teores de carbono orgânico no LVd-a foram submetidos às análises de variância e de regressão, ajustando-se modelos polinomiais de $1^{\circ}, 2^{\circ}$ e $3^{\circ}$ grau. Foi selecionada a equação de regressão de efeito significativo pelo teste F a 5\% de probabilidade e de significado agronômico.

Correlações de Pearson foram feitas para determinar a correlação entre as variáveis de sorção e de dessorção e propriedades dos solos.

\section{Resultados e Discussão}

As estimativas das isotermas de Freundlich ajustadas para a sorção de atrazine nos diferentes solos encontram-se nas Figuras 1 e 2 e Tabela 2. Verifica-se que os coeficientes de determinação das equações de Freundlich foram de $99 \%$, descrevendo adequadamente a sorção de atrazine para todos os solos estudados. Os coeficientes angulares das retas $(1 / \mathrm{n})$ das isotermas de sorção apresentaram valores menores que um, indicando que à medida em que a concentração do herbicida em solução aumentou, a percentagem de herbicida sorvido pelo solo diminuiu. De acordo com a classificação proposta por Giles et al. (1960), essas isotermas se enquadram no tipo$\mathrm{L}$, as quais indicam diminuição dos sítios de sorção com o aumento da concentração.

Os valores de $\mathrm{k}_{\mathrm{f}}$ variaram de 1,14 a 10,30, sendo os maiores valores obtidos para o solo GMd e para os horizontes mais superficiais do LVd-a- Figuras 1 e 2- os quais possuem maiores teores de carbono orgânico (Tabela 1). Esta observação está de acordo com diversos autores, entre eles, Luchini et al. (1984), Sonon \& Schwab (1995), Celis et al. (1997) e Jenks et al. (1998). Os baixos valores de $\mathrm{k}_{\mathrm{f}}$ encontrados para atrazine indicam que este herbicida é de pequena sorção pelos solos estudados (IBAMA, 1996).

No LVd-a observa-se uma diminuição nos valores de $\mathrm{k}_{\mathrm{f}}$ com a profundidade no perfil do solo (Figura 1 e Tabela 2). Este fato deve-se à diminuição do teor de carbono orgânico (Tabela 1). A sorção se correlacionou significativa e positivamente com o teor de carbono orgânico quando analisada isoladamente ou quando todos os solos foram analisados em conjunto (Figura 3 e Tabela 3).

Jenks et al. (1998) encontraram mais atrazine sorvida em amostras de solos nas camadas 0-30 $\mathrm{cm}$ do que em amostras abaixo de 60 $\mathrm{cm}$. Esses autores afirmam que a sorção de atrazine se correlaciona positivamente com a matéria orgânica e negativamente com o pH. A sorção também aumentou com o aumento da matéria orgânica, e diminuiu com o aumento do $\mathrm{pH}$ do solo, mas este fato não ficou evidente nas amostras do LVd-a, possivelmente devido às pequenas variações do $\mathrm{pH}$ com a profundidade. Para o solo LVd-m, o qual apresenta $\mathrm{pH}$ mais elevado do que os outros solos e menor carbono orgânico 

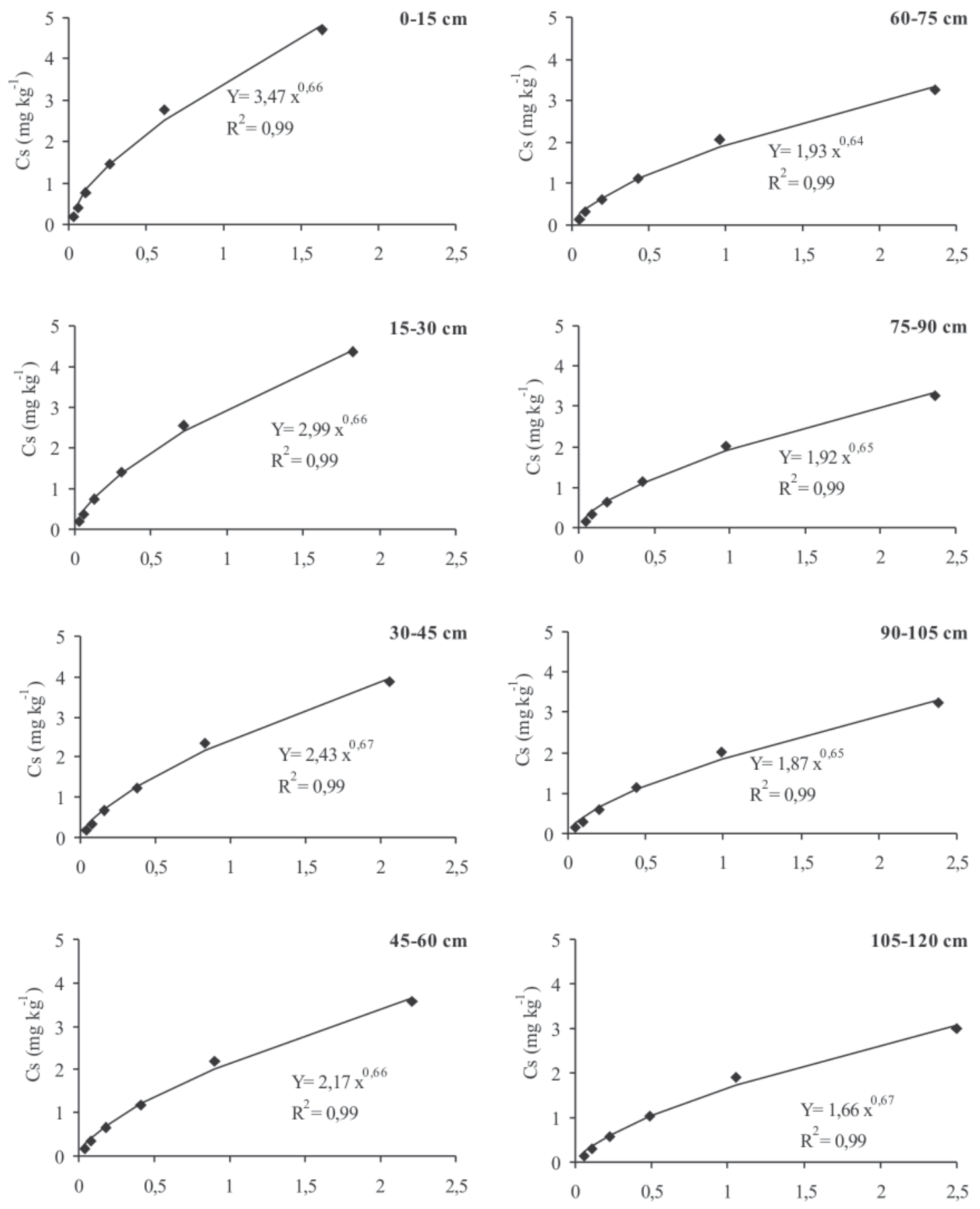

$\mathrm{Ce}\left(\mathrm{mg} \mathrm{L}^{-1}\right)$

$\mathrm{Ce}\left(\mathrm{mg} \mathrm{L}^{-1}\right)$

FIGURA 1. Isotermas de Freundlich ajustadas para descrever a sorção de atrazine nas amostras do perfil do Latossolo Vermelho distrófico, textura argilosa (LVd-a), nas profundidades de 0-120 cm (Cs: concentração sorvida e Ce: concentração em equilíbrio). 

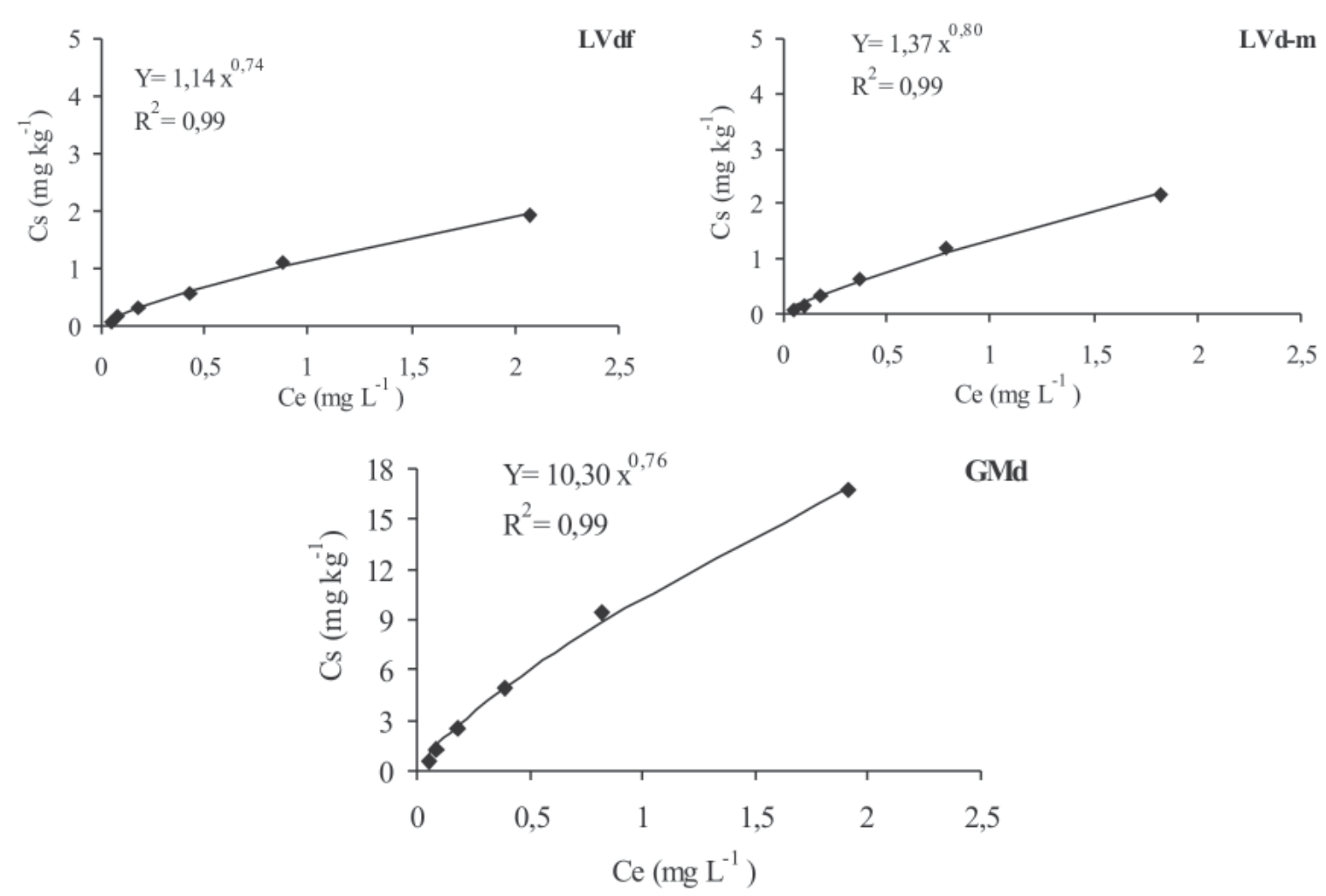

FIGURA 2. Isotermas de Freundlich ajustadas para descrever a sorção de atrazine em amostras do Latossolo Vermelho distroférico (LVdf), Latossolo Vermelho distrófico, textura média (LVd-m) e Gleissolo Melânico distrófico (GMd) coletadas de 0-15 cm de profundidade (Cs: concentração sorvida e Ce: concentração em equilíbrio).

(Tabela 1), esse comportamento também foi observado (Tabela 2). Nos trabalhos de Oliveira Júnior et al. (2001), considerando solos de pHs semelhantes, a sorção de simazine foi claramente proporcional ao teor de carbono orgânico.

Além do maior valor do $\mathrm{pH}$ (Tabela 1) e do $\mathrm{pH}$ do sobrenadante da sorção (Tabela 2) do LVd-m, esse solo possui um menor teor de carbono orgânico e argila, e alto teor de areia, comparado com outros (Tabela 1). Considerando o $\mathrm{pH}$ do sobrenadante desse solo, a maior parte das moléculas de atrazine permaneceu em solução do solo, pois estaria na forma ionizada. A sorção de atrazine foi menor no solo de maior $\mathrm{pH}$. Isso ocorre porque atrazine é uma base fraca e possui pKa 1,7 a $21^{\circ} \mathrm{C}$ (Rodrigues \& Almeida, 1998).
Segundo Oliveira Júnior (1998), à medida em que o pH aumenta, a proporção da forma catiônica em solução diminui, e portanto, a sorção também diminui.

$\mathrm{Na}$ Tabela 3, observa-se que além do carbono orgânico, o $\mathrm{k}_{\mathrm{f}}$ correlacionou-se positiva e significativamente com os teores de capacidade de troca cationica e silte, quando todos os solos foram analisados em conjunto. Nos solos tropicais, altamente intemperizados, onde a fração argila é predominantemente composta por argilas silicatadas e oxídicas, a maior parte da capacidade de troca catiônica é devida à matéria orgânica do solo, o que explica a simultaneidade de significância para as correlações, quando todos os solos foram analisados em conjunto, ou 
TABELA 2. Estimativas das Isotermas de Freundlich, coeficientes de determinação $\left(\mathrm{R}^{2}\right)$ das isotermas e $\mathrm{pH}$ dos sobrenadantes de sorção e dessorção de atrazine nos solos estudados.

\begin{tabular}{lcccccc|ccc}
\hline Classes $^{\text {I- }}$ dos solos & Prof. & \multicolumn{5}{c|}{ Sorção } & \multicolumn{3}{c}{ pH - Dessorção } \\
\cline { 3 - 10 } (cm) & $\mathrm{k}_{\mathrm{f}}$ & $1 / \mathrm{n}$ & $\mathrm{R}^{2}$ & $\mathrm{k}_{\mathrm{foc}}$ & $\mathrm{pH}_{1}$ & $\mathrm{pH}_{2}$ & $\mathrm{pH}_{3}$ & $\mathrm{pH}_{4}$ \\
\hline LVd-a & $0-15$ & 3,47 & 0,66 & 0,99 & 143,98 & 4,40 & 4,42 & 4,46 & 4,63 \\
LVd-a & $15-30$ & 2,99 & 0,66 & 0,99 & 156,54 & 4,17 & 4,20 & 4,22 & 4,29 \\
LVd-a & $30-45$ & 2,43 & 0,67 & 0,99 & 147,88 & 4,14 & 4,14 & 4,15 & 4,21 \\
LVd-a & $45-60$ & 2,17 & 0,66 & 0,99 & 155,71 & 4,15 & 4,15 & 4,16 & 4,22 \\
LVd-a & $60-75$ & 1,93 & 0,64 & 0,99 & 156,91 & 4,16 & 4,16 & 4,17 & 4,22 \\
LVd-a & $75-90$ & 1,92 & 0,65 & 0,99 & 172,97 & 4,18 & 4,17 & 4,18 & 4,24 \\
LVd-a & $90-105$ & 1,87 & 0,65 & 0,99 & 164,04 & 4,19 & 4,18 & 4,19 & 4,23 \\
LVd-a & $105-120$ & 1,66 & 0,67 & 0,99 & 155,14 & 4,18 & 4,19 & 4,19 & 4,23 \\
LVdf & $0-15$ & 1,14 & 0,74 & 0,99 & 70,37 & 4,50 & 4,58 & 4,51 & 4,61 \\
LVd-m & $0-15$ & 1,37 & 0,80 & 0,99 & 201,47 & 6,53 & 6,28 & 6,42 & 6,43 \\
GMd & $0-15$ & 10,30 & 0,76 & 0,99 & 120,75 & 4,52 & 4,50 & 4,61 & 4,66 \\
\hline
\end{tabular}

${ }^{1 /}$ LVd-a, Latossolo Vermelho distrófico, textura argilosa; LVdf, Latossolo Vermelho distroférico; LVd-m, Latossolo Vermelho distrófico, textura média; GMd, Gleissolo Melânico distrófico.

Prof.: Profundidade de amostragem; $\mathrm{k}_{\mathrm{f}}$ : coeficiente de sorção; $1 / \mathrm{n}$ : índice da intensidade da sorção: $\mathrm{k}_{\mathrm{foc}}$ : constante de sorção normalizada para o carbono orgânico; $\mathrm{pH}_{1}: \mathrm{pH}$ do sobrenadante após o ensaio de sorção; $\mathrm{pH}_{2}, \mathrm{pH}_{3}$ e pH $\mathrm{pH}_{4}$ : do sobrenadante após as três dessorções 24,48 e 72 horas, respectivamente.

quando foi analisado somente o perfil do LVd-a. Entretanto, quando a análise de correlação foi realizada somente com o perfil do LVd-a, a correlação com o teor de silte não apresentou efeito significativo. Esse fato demonstra que a análise conjunta de valores de $\mathrm{k}_{\mathrm{f}}$ de diferentes solos pode levar a resultados não satisfatórios. Por isso, é importante fazer uma análise individual de cada solo em termos de sorção, visando identificar separadamente os diversos efeitos que contribuem para a retenção dos herbicidas no solo.

Os valores de $\mathrm{k}_{\text {foc }}$ (Tabela 2) apresentaram variação de 70,37 a 201,47 nas amostras dos solos superficiais, indicando que, além do carbono orgânico, outras propriedades do solo também determinam a sorção de atrazine, resultados esses que são confirmados por Barriuso et al., 1994 e Celis et al., 1997. Segundo Rodrigues \& Almeida (1998) e Graveel \& Turco (2003), o valor médio de $\mathrm{k}_{\text {foc }}$ para atrazine é de 100 . A efetividade relativa do carbono orgânico como uma fase de partição para atrazine é geralmente avaliada por meio da normalização do $\mathrm{k}_{\mathrm{f}}$, observado para o teor de carbono orgânico do solo $\left(\mathrm{k}_{\text {foc }}\right)$. Esse conceito de $\mathrm{k}_{\text {foc }}$ assume que a capacidade de sorção pelo solo é controlada somente 


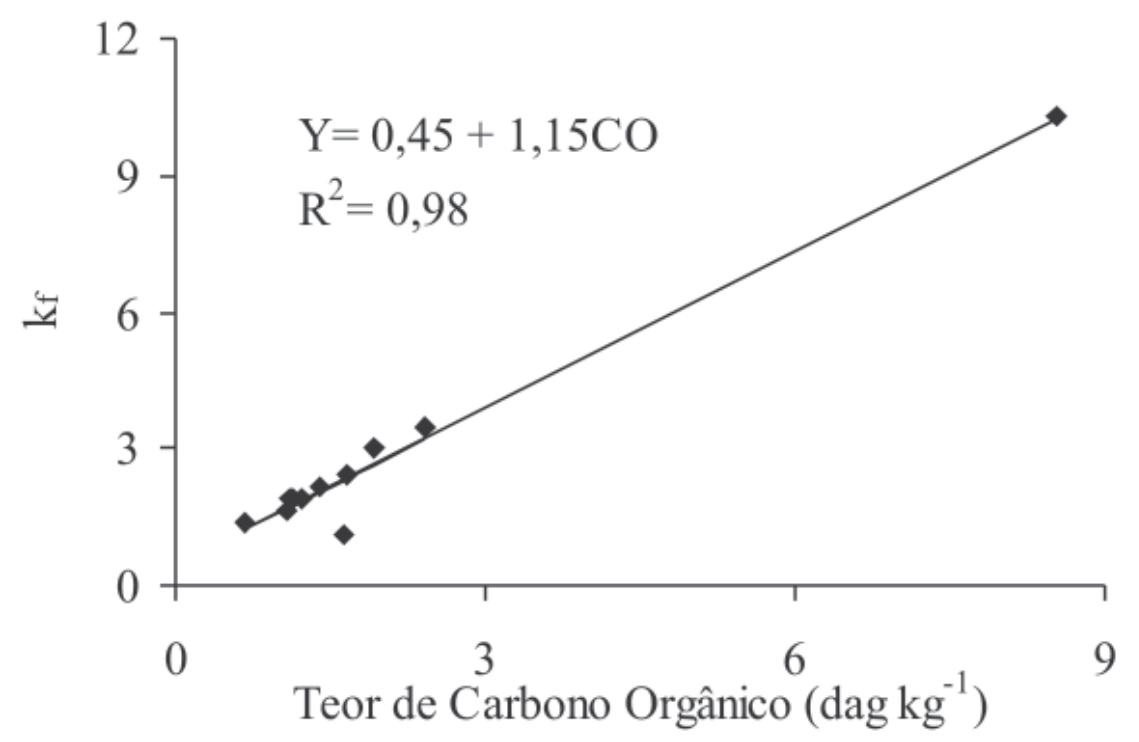

FIGURA 3. Relação entre coeficiente de sorção $\left(\mathrm{k}_{\mathrm{f}}\right)$ para o herbicida atrazine e os teores de carbono orgânico (CO) dos solos estudados.

pelo teor de carbono orgânico. Os valores de $\mathrm{k}_{\text {foc }}$ obtidos dessa forma nesse trabalho não se correlacionaram significativamente com as propriedades dos solos quando todos foram analisados em conjunto. Houve correlação significativa negativa com o teor carbono orgânico $(\mathrm{P}<5 \%)$ quando analisado somente o perfil do LVd-a (Tabela 3).

Considerando o índice de GUS a partir dos valores de meia-vida encontrados por Nakagawa et al. (1995), esse herbicida seria classificado como lixiviável (Figura 4). Resultados semelhantes também foram encontrados por Graveel \& Turco (2003).

Embora a duração da meia-vida no campo para o atrazine possa ser reduzida por fatores como diminuição do $\mathrm{pH}$ do solo (Walker \& Blachlow, 1995 e Nakagawa et al., 1996), elevação da temperatura (Bowmer, 1991) e diminuição do teor de água disponível nos solos (Walker \& Blacklow, 1994 e 1995), este poderia ser classificado como herbicida não-lixiviável em todos os solos, somente se o valor da meia-vida fosse menor que sete dias.
Nas Figuras 5 e 6 estão apresentadas as percentagens acumuladas de dessorção a 24, 48 e 72 horas. Observa-se que esse processo é lento e que a dessorção não é totalmente facilitada com o extrator utilizado $\left(\mathrm{CaCl}_{2}\right)$, mesmo com três sucessivas dessorções, atingindo após 72 horas apenas $23 \%$ para a camada superior $(0-15 \mathrm{~cm})$ e $55 \%$ para a última camada $(105-120 \mathrm{~cm})$ do perfil do LVd-a (Figura 5) e 19\%, 31\% e 44\% para LVdf, LVd e GMd, respectivamente (Figura 6). Isto demonstra que o processo de dessorção é mais lento que o processo de sorção. A liberação pelo solo de apenas parte das moléculas anteriormente sorvidas caracteriza o fenômeno denominado histerese.

Jenks et al. (1998) verificaram uma menor concentração de atrazine dessorvido em amostras de solos após três sucessivas dessorções. Na camada 0-30 cm, encontraram uma concentração de $34 \%$ de atrazine e na camada de 30 a $60 \mathrm{~cm} \mathrm{57 \%}$. Nas amostras do perfil do LVd-a, dados semelhantes foram verificados (Figura 6). 


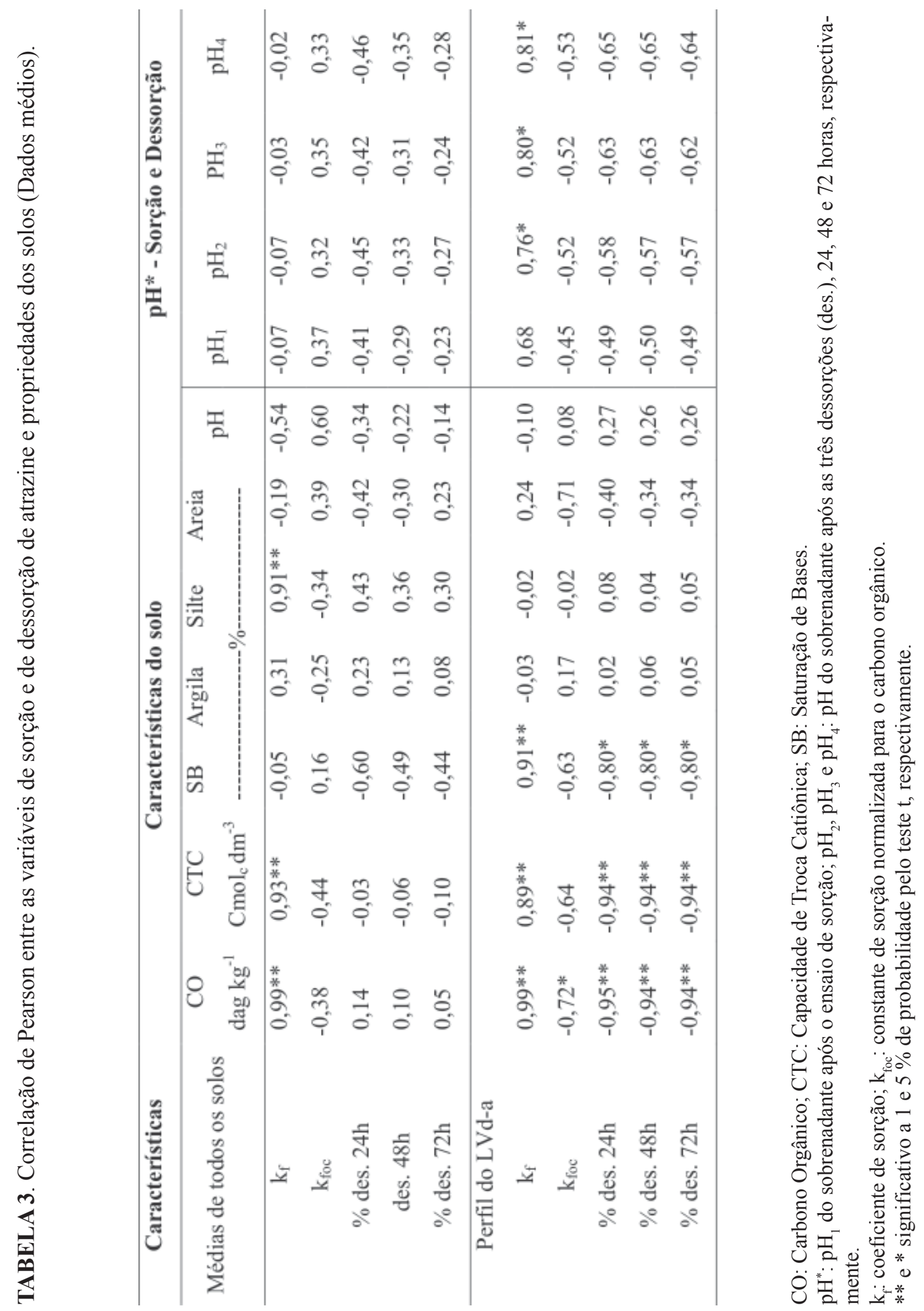

Revista Brasileira de Milho e Sorgo, v.4, n.1, p.14-27, 2005 


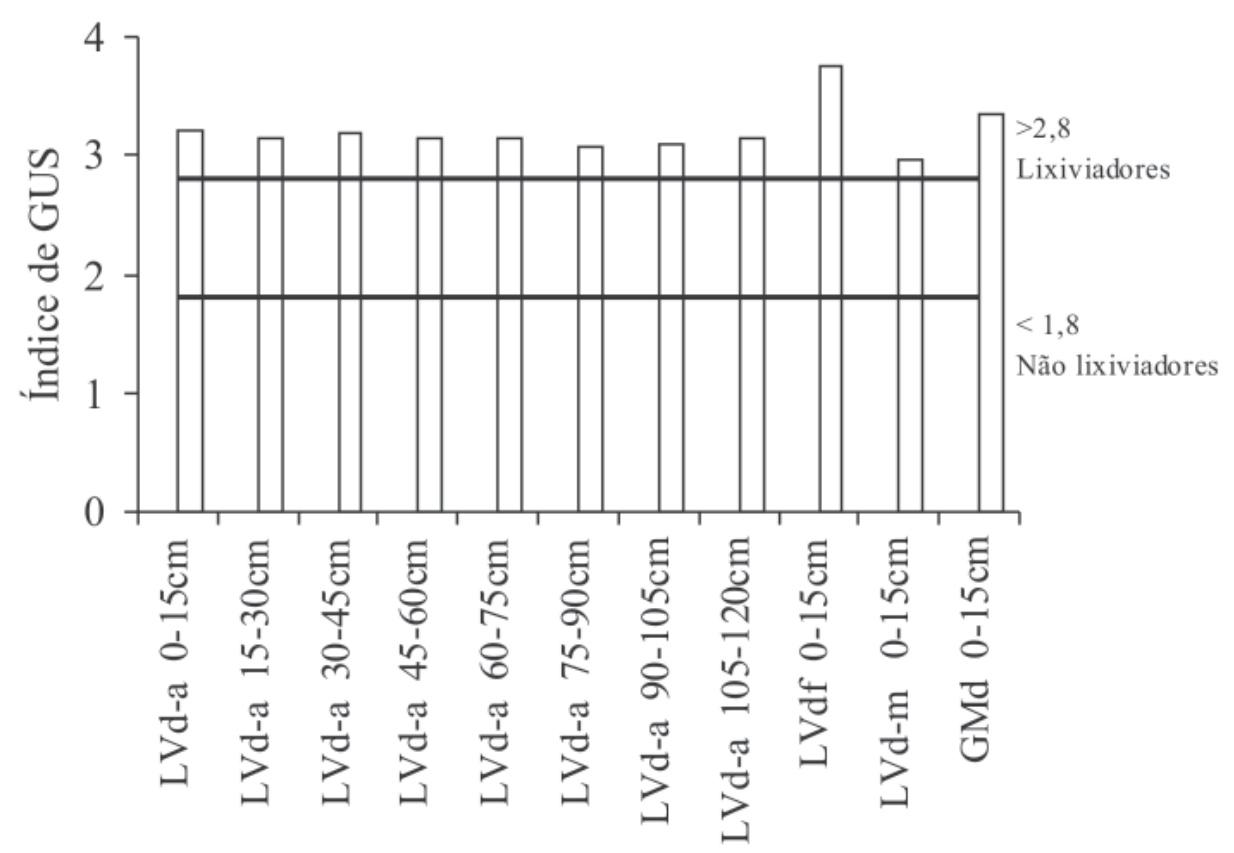

FIGURA 4. Índice de GUS para o herbicida atrazine no Latossolo Vermelho distrófico, textura argilosa (LVda), nas profundidade de: $0-15,15-30,30-45,45-60,60-75,75-90,90-105$ e 105-120 cm e nos Latossolo Vermelho distroférico (LVdf), Latossolo Vermelho distrófico, textura média (LVd-m) e Gleissolo Melânico distrófico $(\mathrm{GMd})$, na profundidade de $0-15 \mathrm{~cm}$.

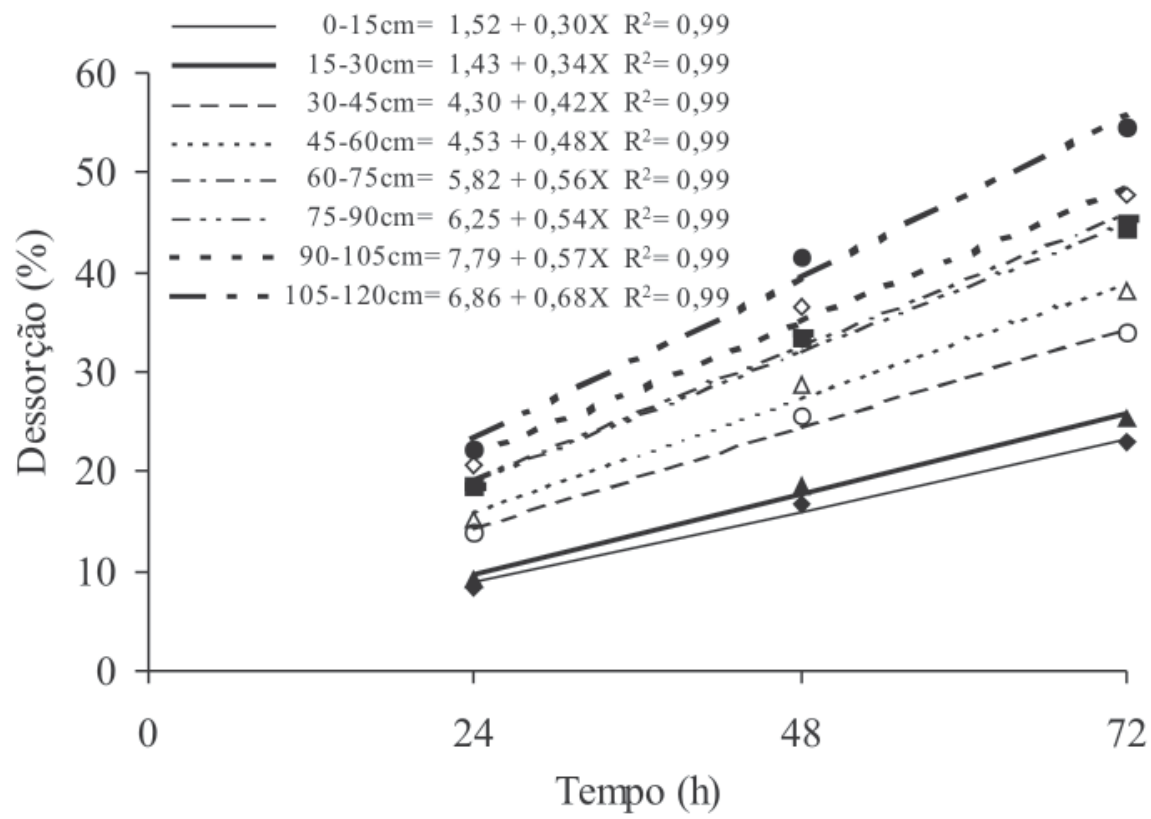

FIGURA 5. Percentagens de dessorção de atrazine no perfil do Latossolo Vermelho distrófico, textura argilosa (LVd-a), nas profundidade de 0-15, 15-30, 30-45, 45-60, 60-75, 75-90, 90-105 e de 105-120 cm em função do tempo. 
Este fato deve-se à diminuição do teor de carbono orgânico com a profundidade do perfil (Tabela 1), uma vez que as dessorções se correlacionaram negativa e significativamente com o teor de carbono orgânico, capacidade de troca catiônica e saturação de bases quando analisadas isoladamente, fato não ocorrido quando todos os solos foram analisados em conjunto (Tabela 3).
A correlação dos valores de $\mathrm{k}_{\mathrm{f}}$ foi significativa e positiva $(\mathrm{P}<5 \%)$ com o $\mathrm{pH}$ do sobrenadante das três dessorções (Tabela 3), quando analisado somente o perfil do LVd-a. Esse fato, possivelmente, pode ser explicado pela pequena variação de $\mathrm{pH}$ do sobrenadante nas camadas 0-15 cm e 15-30 cm (Tabela 2). A relação entre percentagem total de dessorção e teor de carbono orgânico no perfil do LVd-a

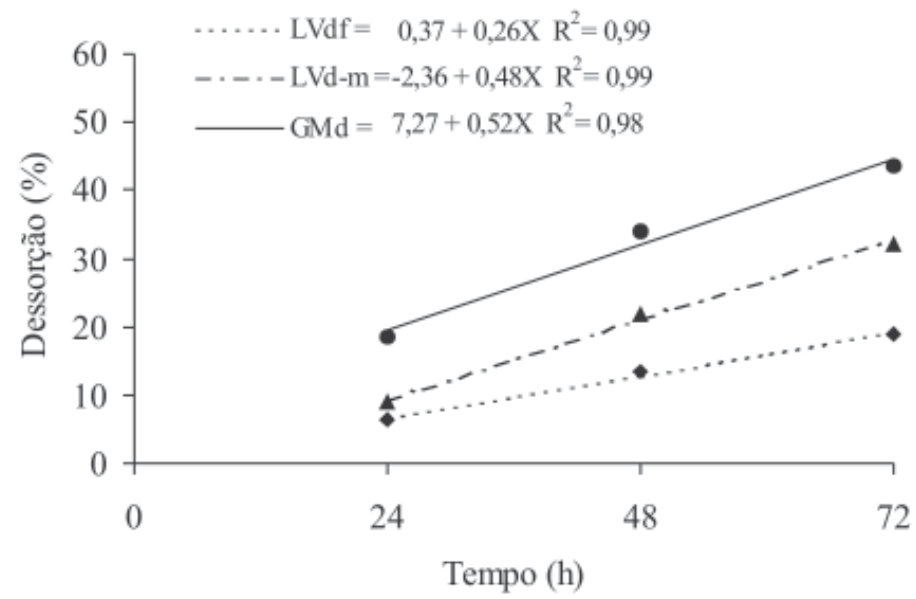

FIGURA 6. Percentagens de dessorção de atrazine nos Latossolo Vermelho distroférico (LVdf), Latossolo Vermelho distrófico, textura média (LVd-m) e Gleissolo Melânico distrófico (GMd), nas profundidades de 0$15 \mathrm{~cm}$ em função do tempo.

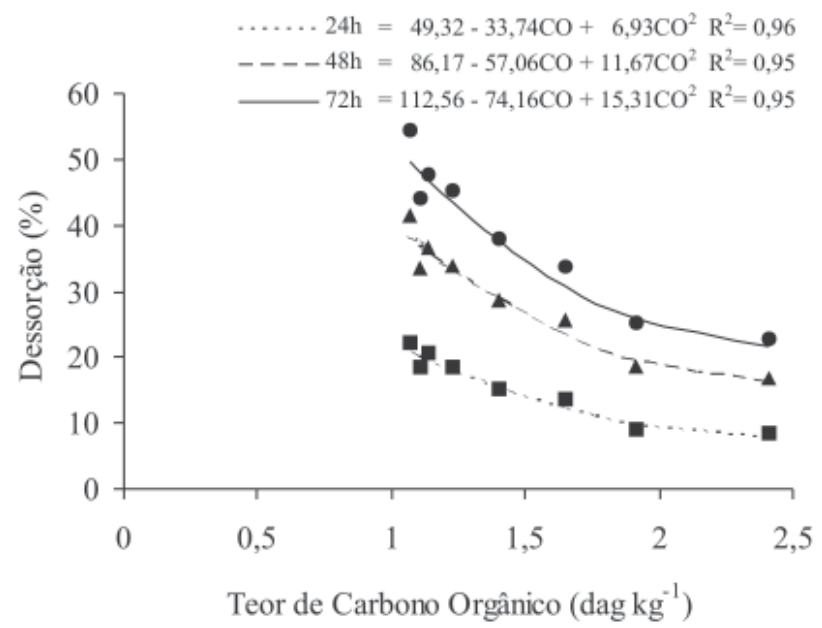

FIGURA 7. Relação entre percentagem total de dessorção de atrazine e o teor de carbono orgânico (CO) no perfil do Latossolo Vermelho distrófico, textura argilosa (LVd-a), considerando dados obtidos das amostras até $120 \mathrm{~cm}$ de profundidade. 
permitiu um bom ajuste de equações de regressão (Figura 7). As equações de regressão de $24 \mathrm{~h}$ e $72 \mathrm{~h}$ devem ser observadas com restrição, uma vez que o desvio também foi significativo.

\section{Conclusões}

Os coeficientes angulares das retas das isotermas de sorção de atrazine, em todos os solos estudados, se enquadraram no tipo-L, as quais indicam diminuição dos sítios de sorção com o aumento da concentração.

Os baixos valores de $\mathrm{k}_{\mathrm{f}}$ encontrados mostram que a atrazine é um herbicida de baixa capacidade de sorção.

Os valores de $\mathrm{k}_{\text {foc }}$ apresentaram correlação significativa com o teor de carbono orgânico quando analisado somente o perfil do LVd-a.

Pela avaliação do potencial de lixiviação conclui-se que a atrazine é um herbicida lixiviado em todos os solos analisados.

O processo de dessorção foi menor que o processo de sorção até 72 horas após a aplicação.

A dessorção é maior em camadas mais profundas no perfil do solo LVd-a, onde o teor de carbono orgânico é menor.

\section{Literatura Citada}

BARRIUSO, E.; LAIRD, D. A.; KOSKINEN, W. C.; DOWDY, D. H. Atrazine desorption from smectites. Soil Science Society American Journal, Madison, v. 58, p. 1632-1638, 1994.

BOWMER, K. H. Atrazine persistence and toxicity in two irrigated soils of Australia. Austalian Journal Soil Residue, Victoria, v. 29, n. 2, p. 339-350, 1991.

CELIS, R.; CONEJOS, J.; HERMOSÍN, M. C.; KOSKINEN, W. C. Sorption-desorption of atrazine and simazine by model soil colloidal components. Soil Science Society American Journal, Madison, v. 61, p. 436-443, 1997.

GILES, C. H.; McEWAN, J. H.; NAKHWA, S. N. et al. Studies in adsorption. Part XI. A system of classification of solution adsorption isoterms, and its use in diagnosis of adsorption mechanisms and in measurement of specific surface areas of solids. Journal Chemistry Society, v. 3, p. 39733993, 1960.

GRAVEEL, J. G.; TURCO, R. F. Soil principles herbicide properties and interactions with soil. In: HERBICIDE action: an intensive course on the activity, selectivity, behavior, and fate of herbicides in plants and soils. West Lafayette. Purdue University, 2003. v.2, p. 667-730.

GUSTAFSON, D. I. Groundwater ubiquity score: a simple method for assessing pesticide leachibility. Environmental Toxicology and Chemistry, Elmsford, v. 8, n. 4, p. 339-357, 1989.

INSTITUTO BRASILEIRO DO MEIO AMBIENTE. Manual de testes para avaliação da ecotoxicidade de agentes químicos, 2. ed. IBAMA, (2ª Revisão, in press), 1996.

JENKS, B. M.; ROETH, F.W., MARTIN, A.R. Influence of surface and subsurface soil properties on atrazine sorption and degradation. Weed Science, Champaign, v.46, p.132-138, 1998.

KHAN, S. E. Pesticides in the soil environment. Amsterdam: Elsevier, 1980.

LATACHANA, A. Note on the effects residual atrazine on succeeding rotation crops. Indian Journal of Weed Science, Haryana, v.19, n. 12, p.111-115, 1987.

LUCHINI, L. C.; HIRATA, R., RUEGG, E. F. Sorção e mobilidade de pesticidas associadas a 
propriedades físico-químicas de solos dos cerrados do estado de São Paulo. Pesquisa Agropecuária Brasileira, Brasília, v. 19, n. 2, p. 157162, 1984.

NAKAGAWA，L. E.; LUCHINI，L. C.; MUSUMECI, M. R.; ANDRÉA, M. M. Comportamento da atrazina em solos brasileiros em condições de laboratório. Pesquisa Agropecuária Brasileira, Brasília, v. 30, p. 471-476, 1995.

NAKAGAWA，L. E.; LUCHINI，L. C.; MUSUMECI, M. R.; MATALLO, M. Behavior of atrazine in soils of tropical zone degradation, mobility and uptake of atrazine residues from soils in a crop rotation system (maize/beans). Journal Environmental Science Health - Part B. Pesticides, Food Contaminants and Agricultural, New York, v. B31, n.2, p. 203-224, 1996.

OLIVEIRA JÚNIOR, R. S. Relação entre propriedades químicas e físicas do solo e sorção, dessorção e potencial de lixiviação de herbicidas. 1998. 83 f. Tese (Doutorado em
Fitotecnia) - Universidade Federal de Viçosa, Viçosa.

OLIVEIRA JÚNIOR, R. S.; KOSKINEN, W. C.; FERREIRA, F. A. Sorption and leaching potencial of herbicides on Brasilian soils. Weed Research, Oxford, v. 41:2, p. 97-110, 2001.

RODRIGUES, B. N.; ALMEIDA, F. L. S. Guia de herbicidas. 4. ed. Londrina: IAPAR, 1998. $648 \mathrm{p}$.

SONON, L. S.; SCHWAB, A. P. Adsorption characteristics of atrazine and alachlor in Kansas soils. Weed Science, Ithaca, v. 43, p. 461-466, 1995.

WALKER, S. R.; BLACKLOW, W. M. Adsorption and degradation of triazine herbicides in soils used for lupin production in Western Australia: Laboratory studies and a simulation model. Australia Journal Soil Residue, Victoria, v. 32, p. 1189-1205, 1994.

WALKER, S. R.; BLACKLOW, W. M. Movement and persistence of triazine herbicides in soil used for lupin production in Western Australia: field measurements and interpretations with the aid of a simulation model. Australia Journal Soil Residue, Victoria, v. 33, p. 773786, 1995. 